\title{
A suggested vision of crisis management of swimming pools
}

"Dr/Samah Mohammed Amin Halawa

Research Introduction \& Problem

Crisis management is considered one of the main elements of any administration's success or failure; as risk accompanies any athletic administration either from the technical or the administrative aspects. It is wrong to think that the leader can fully secure his administration from risks; the leader's responsibility lies in reducing the occurrence of crises or predicting the risks that accompany the activity or the administration.

Crisis management requires quick response and admitting the truth, also challenging the crisis and facing it is always better than escaping, the administration should also admit another truth which is that watch pointers always move forward, and that time never goes back. (10)

Crisis management is nothing but the result of absence of planning, policies or strategies; as administration does not move unless there is a crisis. (2)]

It is necessary for any establishment that is keen on continuing its progress to make a continuous evaluation of all aspects of work in it; as evaluation and its programs became in the modern age relevant to all the operations played by the educational establishments. Because without it, you can't know the reasons of the success achieved or the difficulties you may face, consequently, no development can be done. (1)]

When the crisis occurs, it requires taking the hardest and quickest decisions; the main characteristic of leading the crises is to keep things simple and to ask people to do things or works they are trained on and not to do new and unusual works. (3)]

There are many difficulties face the directors of swimming pools which cause lots of crises, for example,

* teacher in Fencing \& Water Sport Department, Faculty of Physical Education

Assiut Journal For Sport Science Arts 
when a fire occurs in the swimming pools or a drowning or chlorine leaking or electric damage or lighting black out during training, swine flu and its role in reducing practice in the swimming pools or few numbers of activity practices or the crises that may happen in the interior tournaments or any other expected crises.

Therefore, we must put a strategic vision to help administrations, and directors of swimming pools in particular to manage the crisis and to know the reasons behind its occurrence and how to avoid it and manage it using scientific methods and to evaluate it in order to avoid its occurrence again.

\section{Research Aims:}

Research aims to make a suggested vision to manage the crises which swimming pools face through recognizing the following:

1- The reasons of crises in swimming pools.

2- Avoiding crises in swimming pools.

3- The scientific method of crises management in swimming pools.

4- Evaluation methods of administration performance in swimming pools.

\section{Research Questions:}

1- What are the reasons of crises in swimming pools?

2- How to avoid the crises in swimming pools?

3- What is the scientific method of crises management in swimming pools?

4- What are the evaluation methods of management performance in swimming pools?

\section{Research Term:}

Crises Management: is the ability of the decision maker to manage the crisis, before, during and after its occurrence, and to avoid its negative side and benefit from the positive one.

[Mahmoud Abu Samra, Mohammed Eltity, and Faten Ashour (2012), (10)] Research Procedures: First: research Method:

The researcher used the descriptive method "survey study" as it suits the nature of the research.

Second: Research Society \& Sample 


\section{A- Research Society:}

The research society of this study represents "directors - deputy directors administrative staff - rescuers" in the clubs' swimming pools of both governorates of (Cairo - Giza).

\section{B- Research Sample:}

The research sample of this study is represented in "directors - deputy directors administrative staff - rescuers" in the clubs' swimming pools; their number was (101), the researcher chose the research sample deliberately.

Third: Tools of Data Gathering:

A- Scientific References \& Researches:

The researcher reviewed many scientific references and researches and the previous

\section{Table (1)}

The percentage of expert's agreement on questionnaire axes $\mathrm{N}=5$

\begin{tabular}{c|c|c|c}
\hline \hline No. & Axes & $\begin{array}{c}\text { Experts' } \\
\text { opinion } \\
\text { agreement }\end{array}$ & percentage \\
\hline \hline 1 & $\begin{array}{c}\text { Reasons of crises } \\
\text { occurrence in swimming } \\
\text { pools. }\end{array}$ & 5 & $100 \%$ \\
\hline 2 & $\begin{array}{c}\text { Factors of safety \& } \\
\text { security in swimming } \\
\text { pools. }\end{array}$ & 3 & $60 \%$ \\
\hline 3 & $\begin{array}{c}\text { Avoiding crises in } \\
\text { swimming pools. }\end{array}$ & 5 & $100 \%$ \\
\hline \hline
\end{tabular}

Assiut Journal For Sport Science Arts studies that addressed the fields of administration, athletic administration, tests and measurements, and the crises management in the athletic field.

B- Designing the questionnaire form:

The researcher designed a study questionnaire form of "a suggested vision of crises management in swimming pools", the researcher used the steps of questionnaire building according to the rules of scientific research.

C- Knowing the experts' opinions:

The researcher put the suggested phrases in the questionnaire form, then, it was displayed on (5) experts in the field of athletic administration, enclose (2). 
Follow Table (1)

The percentage of expert's agreement on questionnaire axes $\mathrm{N}=5$

\begin{tabular}{c|c|c|c}
\hline \hline No. & Axes & $\begin{array}{c}\text { Experts' } \\
\text { opinion } \\
\text { agreement }\end{array}$ & percentage \\
\hline \hline 4 & $\begin{array}{c}\text { How to prepare for crises } \\
\text { management in } \\
\text { swimming pools. }\end{array}$ & 2 & $40 \%$ \\
\hline 5 & $\begin{array}{c}\text { The scientific method of } \\
\text { crises management in } \\
\text { swimming pools. }\end{array}$ & 5 & $100 \%$ \\
\hline 6 & $\begin{array}{c}\text { The evaluation methods } \\
\text { of crises management in } \\
\text { swimming pools. }\end{array}$ & 5 & $100 \%$ \\
\hline \hline
\end{tabular}

Table (1) show that the axes of the questionnaire "a suggested vision of crises management in swimming pools" have been determined, its relative importance percentage was between $(40 \%$ - $100 \%$ ) though the results of the experts' poll form, the researcher approved the percentage $(100 \%)$ to determine the preliminary axes of the questionnaire form.

Then, the researcher put the phrases of the open questionnaire form; its number was (51) phrases distributed on (4) axes, and she displayed the phrases of each axis on the experts; enclose (3) to check the reasonable validity and that the suggested phrases are suitable for each axis, and how far are the suggested phrases formation suitable for the axis they belong, and how far can we omit, adjust or add other phrases. Through knowing the experts' opinions, the researcher could determine the relative importance of the experts' agreement,

The next table clarifies the number of the 2 questionnaires phrases in their preliminary shape and the number of the omitted phrases and their figures according to knowing the experts' opinions. 
Tables (2)

The number of questionnaire's phrases in its preliminary shape and the number of the omitted phrases and its figures according to the percentage of experts' opinions

\begin{tabular}{c|c|c|c|c|c}
\hline \hline No. & Axes & $\begin{array}{c}\text { Number of } \\
\text { axis's } \\
\text { preliminary } \\
\text { phrases }\end{array}$ & $\begin{array}{c}\text { Number } \\
\text { of } \\
\text { omitted } \\
\text { phrases }\end{array}$ & $\begin{array}{c}\text { Number } \\
\text { of initial } \\
\text { phrases }\end{array}$ & $\begin{array}{c}\text { figures } \\
\text { of } \\
\text { omitted } \\
\text { phrases }\end{array}$ \\
\hline \hline 1 & $\begin{array}{c}\text { Reasons of } \\
\text { crises } \\
\text { occurrence in } \\
\text { swimming } \\
\text { pools. }\end{array}$ & 10 & 1 & 14 & 8 \\
\hline 2 & $\begin{array}{c}\text { Avoiding crises } \\
\text { in swimming } \\
\text { pools }\end{array}$ & $1 r$ & 1 & 11 & 10 \\
\hline 3 & $\begin{array}{c}\text { The scientific } \\
\text { method of crises } \\
\text { management in } \\
\text { swimming pools }\end{array}$ & $1 r$ & 1 & 11 & 6 \\
\hline 4 & $\begin{array}{c}\text { Evaluation } \\
\text { methods of } \\
\text { administration } \\
\text { performance in } \\
\text { swimming pools }\end{array}$ & $1 r$ & - & 12 & - \\
\hline Total & $\mathbf{5 1}$ & $\mathbf{0 1}$ & $\mathbf{4 8}$ & \\
\hline \hline
\end{tabular}

Table (2) shows the number of questionnaire's phases in its preliminary shape, the number of the omitted phrases from each axis, the number of the axis's phrases after excluding the omitted phrases and the figures of the omitted phrases according to the percentage of experts' opinions.

Thus, the total number of questionnaire's phrases 'the suggested vision of crisis management in swimming pools" became (48) phrases. Fourth: the Scientific Transactions of the questionnaire:

1- The studies form validity:

(A) the judges' validity (the reasonable validity):

The researcher used the judges' validity (the reasonable validity), as the form in its preliminary shape enclose (3) was displayed on (5) experts (judges), and the researcher 
considered the percentage of experts' agreement on the questionnaire's phrases a proof of its validity.

(B) Validity of internal consistency:

The researcher also calculated the validity of the studied questionnaire form through using the method of internal consistency validity. As the researcher calculated the value of correlation coefficients between the degree of each phrase aside, and the total degree of the questionnaire, and also calculated the value of the correlation coefficients of each phrase aside, and the total degree of each axis to which it belongs. All of this was done after applying the questionnaire form on the study pilot sample; tables (3, 4, and 5) show that.

Table (3)

The correlation coefficients between the total scores of each axis and the total degree of the questionnaire $(N=20)$

\begin{tabular}{c|c|c}
\hline \hline No. & Axes & $\begin{array}{c}\text { Correlation } \\
\text { coefficient and } \\
\text { its significance }\end{array}$ \\
\hline \hline 1 & $\begin{array}{c}\text { Reasons of crises occurrence in } \\
\text { swimming pools. }\end{array}$ & $* .991$ \\
\hline 2 & Avoiding crises in swimming pools & $* .997$ \\
\hline 3 & $\begin{array}{c}\text { The scientific method of crises } \\
\text { management in swimming pools }\end{array}$ & $* .997$ \\
\hline 4 & $\begin{array}{c}\text { Evaluation methods of administration } \\
\text { performance in swimming pools }\end{array}$ & $* .999$ \\
\hline \hline
\end{tabular}

$*(\mathrm{R})$ table value at morale level $(0.05)$, freedom degree $(18)=(0.444)$. Table (3) shows that the The researcher found the values of correlation coefficients are significant at morale level (0.05), as the values of correlation coefficient were between (0.999-0.996).

2-The Reliability of the studied form: reliability coefficient of the questionnaire's axes; their number was (4) axes and the number of their phrases was (44); using two methods: the method of half segmentation of the study pilot sample responses on the questionnaire using the Spearman \& Brown 
equation to find the correlation coefficient between the even phrases and odd phrases, and also to find the reliability using the Cronbach's alpha coefficient.

(a) Reliability using Cronbach's alpha coefficient: The value of Cronbach's alpha coefficient of the phrases of questionnaire "crises management in swimming pools"; which its number was (44), the value was (0.986).

*the Value of (Cronbach's alpha coefficient) of the questionnaire $=(\mathbf{0 . 9 8 6})$.

Table (4) shows the (Cronbach`s alpha coefficient) of the (questionnaire's) phrases and all of them are significant, as its (Cronbach`s alpha coefficient) was (0.987) which are insignificant values; because it is bigger than the (Cronbach`s alpha coefficient) of the questionnaire which requires omitting them as they affect the reliability of the questionnaire. The rest of the questionnaire's phrases were significant as the (Cronbach`s alpha coefficient) value of these phrases were between $(0.985-0.982)$; which is less than the (Cronbach`s alpha coefficient) value of the questionnaire which indicates its reliability.

\section{Tables (5)}

\section{Reliability coefficient using Cronbach`s alpha of the} questionnaire's axes phrases

\begin{tabular}{c|c|c}
\hline \hline No. & axes & $\begin{array}{c}\text { (Cronbach`s } \\
\text { alpha } \\
\text { coefficient) }\end{array}$ \\
\hline \hline$r$ & $\begin{array}{c}\text { Reasons of crises occurrence in swimming } \\
\text { pools. }\end{array}$ & $* .9 \leq r$ \\
\hline$r$ & Avoiding crises in swimming pools & $* .90 \mathrm{r}$ \\
\hline$r$ & $\begin{array}{c}\text { The scientific method of crises management } \\
\text { in swimming pools }\end{array}$ & $* .9 \mathrm{rV}$ \\
\hline$\varepsilon$ & $\begin{array}{c}\text { Evaluation methods of administration } \\
\text { performance in swimming pools }\end{array}$ & $* .9 \mathrm{r} r$ \\
\hline \hline
\end{tabular}

* the value of Cronbach`s alpha coefficient of the questionnaire $=(0.986)$.

Table (5) shows the questionnaire as a whole, and Cronbach`s alpha coefficient all of them are significant; as between all the axes and the the value of Cronbach`s alpha 
coefficient of the axes was between $(0.953,0.936)$ which is smaller than the value of Cronbach`s alpha coefficient of the questionnaire (0.986) which indicates its reliability.

(a)Reliability using half segmentation:

The researcher found the reliability coefficient of the questionnaire's phrases; its number (44) using the method of half segmentation of the study pilot sample responses using Spearman \& Brown equation to find the correlation coefficient between the even and the odd phrases.

\section{Table (6)}

The reliability of the half segmentation of the measurement

\begin{tabular}{c|c|c|c|c|c|c}
\hline \hline Questionnaire & \multicolumn{2}{|c|}{ Odd phrases } & \multicolumn{2}{|c|}{ Even phrases } & Correlation & Table \\
coefficient & "R" \\
\hline \hline $\begin{array}{c}\text { Crises } \\
\text { management in } \\
\text { swimming pools }\end{array}$ & 101.80 & 28.38 & 98.40 & 27.00 & $* 0.995$ & 0.444 \\
\hline
\end{tabular}

Table (6) shows that there is a statistical significant correlation between the measurement phrases as a whole. This indicates the measurement's reliability.
The next table shows the number of the two questionnaires phrases in their final shape and the number of the omitted phrases and their figures according to the experts' opinions.

Table (7)

Number of questionnaire's phrases in their final shape and the number of the omitted phrases and their figures after performing the scientific transactions "validity - reliability"

\begin{tabular}{c|c|c|c|c|c}
\hline \hline No. & Axes & $\begin{array}{c}\text { Number } \\
\text { of axis } \\
\text { phrases }\end{array}$ & $\begin{array}{c}\text { Number } \\
\text { of } \\
\text { omitted } \\
\text { phrases }\end{array}$ & $\begin{array}{c}\text { Final } \\
\text { Number } \\
\text { of axis } \\
\text { phrases }\end{array}$ & $\begin{array}{c}\text { Figures } \\
\text { of } \\
\text { omitted } \\
\text { phrases }\end{array}$ \\
\hline \hline, & $\begin{array}{c}\text { Reasons of crises } \\
\text { occurrence in } \\
\text { swimming pools. }\end{array}$ & $1 \varepsilon$ & 1 & $1 r$ & 11 \\
\hline$r$ & $\begin{array}{c}\text { Avoiding crises in } \\
\text { swimming pools }\end{array}$ & 11 & 1 & 1 & $\wedge$ \\
\hline \hline
\end{tabular}

Assiut Journal For Sport Science Arts 
Table (7)

Number of questionnaire's phrases in their final shape and the number of the omitted phrases and their figures after performing the scientific transactions "validity - reliability"

\begin{tabular}{|c|c|c|c|c|c|}
\hline No. & Axes & $\begin{array}{c}\text { Number } \\
\text { of axis } \\
\text { phrases }\end{array}$ & $\begin{array}{c}\begin{array}{c}\text { Number } \\
\text { of } \\
\text { omitted } \\
\text { phrases }\end{array} \\
\end{array}$ & $\begin{array}{c}\text { Final } \\
\text { Number } \\
\text { of axis } \\
\text { phrases }\end{array}$ & $\begin{array}{c}\begin{array}{c}\text { Figures } \\
\text { of } \\
\text { omitted } \\
\text { phrases }\end{array} \\
\text { phises }\end{array}$ \\
\hline r & $\begin{array}{l}\text { The scientific method of } \\
\text { crises management in } \\
\text { swimming pools }\end{array}$ & 11 & 1 & 1. & $\varepsilon$ \\
\hline$\varepsilon$ & $\begin{array}{c}\text { Evaluation methods of } \\
\text { administration performance } \\
\text { in swimming pools }\end{array}$ & IT & 1 & 11 & 7 \\
\hline & Total & 48 & $\varepsilon$ & 44 & \\
\hline \multicolumn{3}{|c|}{$\begin{array}{l}\text { Table (7) shows the } \\
\text { number of the questionnaire's } \\
\text { phrases in its final shape, the } \\
\text { number of the omitted phrases } \\
\text { from each axis, the number of } \\
\text { the axis phrases after excluding } \\
\text { the omitted phrases and the } \\
\text { figures of the omitted phrases } \\
\text { according to the percentage of } \\
\text { experts' opinions. } \\
\text { - Thus, the total number of the }\end{array}$} & \multicolumn{3}{|c|}{$\begin{array}{l}\text { suggested vision of crises } \\
\text { management in swimming } \\
\text { pools" became (44) phrases. } \\
\text { Eighth: Display \& Discussion } \\
\text { of Results: } \\
\text { First: Display of Results: } \\
\text { 1- Displaying the results of the } \\
\text { study sample responses to the } \\
\text { axis phrases "reasons of crises } \\
\text { occurrence in swimming } \\
\text { pools". }\end{array}$} \\
\hline
\end{tabular}

final questionnaire phrases "a

\section{Table (8)}

Repetition, percentage, (ca2) value and phrases order of the study sample responses to the axis phrases "reasons of crises occurrence in swimming pools" $\mathrm{N}=81$

\begin{tabular}{|c|c|c|c|c|c|c|c|c|c|c|c|}
\hline \multirow[t]{2}{*}{ No. } & \multirow[t]{2}{*}{ Phrases } & \multicolumn{2}{|l|}{ Agree } & \multicolumn{2}{|c|}{ To some extent } & \multicolumn{2}{|l|}{ disagree } & \multirow{2}{*}{$\begin{array}{l}\text { Estimated } \\
\text { total }\end{array}$} & \multirow{2}{*}{$\begin{array}{l}\text { Relative } \\
\text { weight }\end{array}$} & \multirow[t]{2}{*}{$\mathrm{Ca} 2$} & \multirow[t]{2}{*}{ order } \\
\hline & & repetition & $\%$ & Repetition & $\%$ & repetition & $\%$ & & & & \\
\hline \multicolumn{12}{|c|}{ ol admi } \\
\hline$a-$ & $\begin{array}{l}- \\
\text { technical teams }\end{array}$ & $\varepsilon_{0}$ & 0.04 & 9 & v. $\& 1$ & $r$. & $r v_{.} \cdot \varepsilon$ & $1 \leqslant v$ & $7 . \leqslant 9$ & *YA.TV & rv \\
\hline b- & $\begin{array}{l}-\quad \text { Athletic } \\
\text { activity administration }\end{array}$ & \& & or..99 & v & A.T & $r$ & $r \Lambda, r v$ & 10. & $\pi . v r$ & $* Y \leqslant . \wedge q$ & צ \\
\hline c- & $\begin{array}{l} \\
\text { activity administration }\end{array}$ & r. & r $r .79$ & ir & $\mid \leq . \wedge 1$ & $\varepsilon q$ & $7 . \leqslant 9$ & 191 & vA.. & $* \curlyvee \wedge . \cdot \vee$ & 1 \\
\hline
\end{tabular}


Follow Table (8)

Repetition, percentage, (ca2) value and phrases order of the study sample responses to the axis phrases "reasons of crises occurrence in swimming pools" $\mathrm{N}=81$

\begin{tabular}{|c|c|c|c|c|c|c|c|c|c|c|c|}
\hline \multirow[t]{2}{*}{ No. } & \multirow[t]{2}{*}{ Phrases } & \multicolumn{2}{|l|}{ Agree } & \multicolumn{2}{|c|}{ To some extent } & \multicolumn{2}{|l|}{ disagree } & \multirow{2}{*}{$\begin{array}{l}\text { Estimated } \\
\text { total }\end{array}$} & \multirow{2}{*}{$\begin{array}{l}\text { Relative } \\
\text { weight }\end{array}$} & \multirow[t]{2}{*}{$\mathrm{Ca} 2$} & \multirow[t]{2}{*}{ order } \\
\hline & & repetition & $\%$ & Repetition & $\%$ & repetition & $\%$ & & & & \\
\hline \multicolumn{12}{|c|}{ ck of sufficient scientific qualification of workers in the swimming pool: } \\
\hline $\mathrm{a}-$ & $\begin{array}{l}\text { - Maintenance } \\
\text { workers. }\end{array}$ & $\leqslant 1$ & $0 . . \mathrm{Tr}$ & $\wedge$ & $৭ . \wedge \wedge$ & rr & ra.01 & 10r & ד.9 & $*$ * 1.07 & r. \\
\hline $\mathrm{b}-$ & Rescue. & $\leqslant$ & $0 . .7 r$ & 9 & 11.11 & ri & $r \wedge . r v$ & lor & $7 r .00$ & $* 19 . \wedge 0$ & YI \\
\hline$c^{-}$ & Coaches. & $r \leq$ & ra.ir & $1 \leqslant$ & IV.rA & $\varepsilon r$ & or.. 9 & $|1|$ & $V \leqslant . \leqslant 9$ & $* 1 \% . \cdot V$ & $r$ \\
\hline \multicolumn{12}{|c|}{ sis occurs: } \\
\hline$a-$ & $\begin{array}{l}\text { - Absence of } \\
\text { rescuers. }\end{array}$ & ؛ & or.. 9 & 7 & $v . \leqslant 1$ & rr & $r 9.01$ & 101 & 7r.1ะ & *Yษ.V & rr \\
\hline$b-$ & $\begin{array}{l}- \\
\text { cuts. }\end{array}$ & rr & rV.17 & rq & $\leqslant \Lambda .10$ & $r$. & $r \leq .79$ & 17. & $10 . \wedge \varepsilon$ & $* \Lambda . \cdot \vee$ & 1. \\
\hline$c^{-}$ & $\begin{array}{l}\text { - Cold } \\
\text { weather. }\end{array}$ & ir & $1 \leqslant . \wedge 1$ & 00 & 7v.q. & $1 \leq$ & IV.YA & $17 \varepsilon$ & $7 V . \leqslant 9$ & $* \leq r .7 r$ & $v$ \\
\hline$d-$ & $\begin{array}{l}\text { - A crisis with } \\
\text { the members. }\end{array}$ & 11 & Yr.YY & $\leqslant 0$ & 00.04 & 11 & YY.YY & 18 & 8.78 & $* 1 \wedge .$. & $\wedge$ \\
\hline$e^{-}$ & Other. & $r$. & $r \leq .79$ & 01 & 84.94 & 1. & ir.ro & lor & $7 r .00$ & *rr.^• & ri \\
\hline \multicolumn{12}{|c|}{ 4- Parents interference in matters of the swimming pool: } \\
\hline a- & $\begin{array}{l}\text { - Pool } \\
\text { administration. }\end{array}$ & rv & $\leqslant 0.71$ & 1. & 1T.ro & $r \varepsilon$ & $\leqslant 1.91$ & 109 & $r 0 . \leqslant T$ & *1T.r & 11 \\
\hline$b-$ & Coaches. & rr & $r 9.01$ & $1 \varepsilon$ & IV.rA & ro & $\{r . r \mid$ & 170 & $7 v .9$. & $* 9.07$ & 7 \\
\hline $\mathrm{c}^{-}$ & Rescuers. & 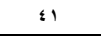 & $0 ., \mathrm{Tr}$ & 1. & $14 . \mathrm{rog}$ & $r$. & $r v . \cdot \varepsilon$ & 101 & 7r.1ะ & $* 1 \wedge . r \cdot$ & rr \\
\hline$d-$ & Other. & $r$. & $r \leq .79$ & $\leqslant V$ & $0 \Lambda_{.} \cdot r$ & $1 \leqslant$ & IV.rA & 107 & $T \leq . Y$. & *rr.^q & ir \\
\hline \multicolumn{12}{|c|}{ 5- Lack of regulations: } \\
\hline$a-$ & $\begin{array}{l}\text { - Being on } \\
\text { the swimming pool. }\end{array}$ & ro & $\{r . r \mid$ & $1 \leq$ & IV.rA & rr & $r 9.01$ & 109 & $r 0 . \leqslant T$ & $* 9.07$ & 11 \\
\hline$b-$ & $\begin{array}{l}\text { Dealing with } \\
\text { the coaches. }\end{array}$ & r\& & r9.7r & rr & rv.17 & ro & $\{r . r \mid$ & IVT & $v 1.19$ & r.ir & • \\
\hline $\mathrm{c}-$ & $\begin{array}{l}\text { Dealing with } \\
\text { the workers. }\end{array}$ & 11 & Yr.YY & 01 & 84.97 & ir & $1 \leq . \wedge 1$ & 107 & $T \leq .$. & *r. & ir \\
\hline$d-$ & $\begin{array}{l}\text { Dealing with } \\
\text { the rescuers. }\end{array}$ & $\varepsilon r$ & or.. 9 & 1 & V. $\leqslant 1$ & rr & $r 9.01$ & 101 & $7 r .1 \leq$ & *ห५.V & rr \\
\hline $\mathrm{e}^{-}$ & Other. & r. & $r \leq .79$ & $\leq V$ & $0 \Lambda . \cdot r$ & $1 \varepsilon$ & IV.rA & 107 & Iะ. . & 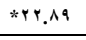 & 14 \\
\hline 6 & $\begin{array}{l}\text { Lack of periodical } \\
\text { maintenance of equipments } \\
\text { and machines of the } \\
\text { swimming pool. }\end{array}$ & 37 & 45.68 & 14 & 17.28 & 30 & 37.04 & 155 & 63.79 & ${ }^{*} 10.30$ & 17 \\
\hline 7 & $\begin{array}{l}\text { The pool's capacity } \\
\text { exceeds the volume of } \\
\text { activities used in it. }\end{array}$ & 24 & r9.7r & rq & $\varepsilon \wedge .10$ & 11 & YY.YY & 107 & $T \leq . Y$. & $* \Lambda .7 \vee$ & ir \\
\hline 8 & $\begin{array}{lr}\text { Lack of } & \text { sufficient } \\
\text { knowledge } & \text { of } \\
\text { maintenance } & \text { systems } \\
\text { and methods. } & \end{array}$ & 37 & $\varepsilon 0.71$ & $1 \leq$ & IV.rA & $r$. & $r v . \cdot \varepsilon$ & 100 & $87 . v 9$ & $* 1 \cdot . r$. & IV \\
\hline
\end{tabular}


Follow Table (8)

Repetition, percentage, (ca2) value and phrases order of the study sample responses to the axis phrases "reasons of crises occurrence in swimming pools" $\mathrm{N}=81$

\begin{tabular}{|c|c|c|c|c|c|c|c|c|c|c|c|}
\hline \multirow[t]{2}{*}{ No. } & \multirow[t]{2}{*}{ Phrases } & \multicolumn{2}{|l|}{ Agree } & \multicolumn{2}{|c|}{ To some extent } & \multicolumn{2}{|l|}{ disagree } & \multirow{2}{*}{$\begin{array}{l}\text { Estimated } \\
\text { total }\end{array}$} & \multirow{2}{*}{$\begin{array}{l}\text { Relative } \\
\text { weight }\end{array}$} & \multirow[t]{2}{*}{$\mathrm{Ca} 2$} & \multirow[t]{2}{*}{ order } \\
\hline & & repetition & $\%$ & Repetition & $\%$ & repetition & $\%$ & & & & \\
\hline 9 & $\begin{array}{l}\text { Not following the } \\
\text { scientific method of } \\
\text { water sterilization } \\
\text { inside the swimming } \\
\text { pool. }\end{array}$ & 41 & $0 . .7 r$ & 7 & V. $\leqslant 1$ & $r \leq$ & $\leqslant 1.91$ & 100 & 7r.va & $* y 0 . \leqslant 1$ & iv \\
\hline 10 & $\begin{array}{l}\text { Lack of first aid official } \\
\text { on the swimming pool. }\end{array}$ & 34 & $\leqslant 1.91$ & it & 17.0 & $r \leq$ & $\leqslant 1.91$ & 175 & 77.78 & $* 1 \cdot . \wedge 9$ & $\wedge$ \\
\hline 11 & $\begin{array}{l}\text { Using the swimming } \\
\text { pool by many people in } \\
\text { the same time. }\end{array}$ & 26 & r..l. & $\leq 9$ & $7 . .59$ & 7 & V.\&1 & $1 \leqslant r$ & $0 \wedge . \leqslant \leq$ & * & re \\
\hline 12 & $\begin{array}{l}\text { Lack of permanent } \\
\text { security guards on the } \\
\text { swimming pool to ban } \\
\text { harassments or any } \\
\text { struggles that may } \\
\text { happen. }\end{array}$ & 22 & rV.17 & 11 & rY.rY & $\leqslant 1$ & $0 . .74$ & $\mid 11$ & $V \leqslant . \leqslant 9$ & $* 11.19$ & $r$ \\
\hline 13 & $\begin{array}{l}\text { Taking long time to do } \\
\text { maintenance works } \\
\text { without another } \\
\text { alternative or a definite } \\
\text { time to finish these } \\
\text { works. }\end{array}$ & 24 & 29.63 & 18 & 22.22 & 39 & 48.15 & 177 & 72.84 & 8.67 & 4 \\
\hline
\end{tabular}

Ca2 table value at morale level $(0.05)$ and freedom degree $(2)=(5.991)$

Table (8) shows that

(Ca2) calculated value of study sample responses to axis phrases "reasons of crises occurrence in swimming pools" were all statistically significant at morale level (0.05), except the phrase number $(5-b)$ where the $(\mathrm{Ca} 2)$ calculated value was (3.63) which is statistically insignificant. As (Ca2) calculated value was between (34.30 - 8.07), and the relative percentage was between $(78.60 \%$ - $58.44 \%)$, as the phrases numbered (1A, 1B, 2A, 2B, 3A, 4c, 5 D) came with high agreement percentage between $(50.62 \%$ $55.56 \%$ ).

The researcher's study assured the lack of coordination between the swimming pool administration and the athletic activity administration by percentage $(09.53 \%)$, this contradicts with the study of (Mahmoud Abu Samra, Mohamed Eltity, and 
Faten Ashour 2012) (10) where they have an open communication system between the department administration, the students and the crises team during the crisis; as coordination and communication are necessary to solve the crises and lack of both of them or even their moderate existence is one of the reasons of crises occurrence in swimming pools. The researcher also sees that one of the reasons behind the crises occurrence is the lack of sufficient qualifying of maintenance and rescue workers in particular, as half of the study sample confirmed that by $(50.62 \%)$, also during

Table (9)

Repetition, percentage, Ca2 value, and phrases order of study sample responses to axis phrases "avoiding crises in swimming pools" $(\mathrm{N}=\mathbf{8 1})$

\begin{tabular}{|c|c|c|c|c|c|c|c|c|c|c|c|}
\hline \multirow[t]{2}{*}{ No. } & \multirow[t]{2}{*}{ Phrases } & \multicolumn{2}{|l|}{ agree } & \multicolumn{2}{|c|}{ To some extent } & \multicolumn{2}{|l|}{ Disagree } & \multirow{2}{*}{$\begin{array}{l}\text { Estimated } \\
\text { total }\end{array}$} & \multirow{2}{*}{$\begin{array}{l}\text { Relative } \\
\text { weight }\end{array}$} & \multirow[t]{2}{*}{$\mathrm{Ca} 2$} & \multirow[t]{2}{*}{ order } \\
\hline & & repetition & $\%$ & repetition & $\%$ & Repetition & $\%$ & & & & \\
\hline \multicolumn{12}{|c|}{ There is an instructions and guidance booklet of the swimming pool for the following: } \\
\hline a- & *employees. & ri & $r \leqslant .0 V$ & rᄉ & $r \leqslant .0 V$ & ro & $r \cdot . \wedge 7$ & 170 & $7 V .9$. & $\because r$ & $r$ \\
\hline$b-$ & "coaches. & or & $70 . \leqslant T$ & KI & ro.9r & V & $1.7 \varepsilon$ & $r \cdot \lambda$ & 10.7. & $* \leq 1.19$ & 11 \\
\hline $\mathrm{c}-$ & "rescuers. & $7 \leqslant$ & $v 9 . \cdot 1$ & 9 & 11.11 & $\wedge$ & $9 . \wedge 1$ & YIA & 19.1 & $* \vee\urcorner . \cdot \vee$ & $\wedge$ \\
\hline d- & *Members. & 07 & $79.1 \leq$ & 17 & 19.10 & 9 & 11.11 & $r \cdot q$ & $17 . \cdot 1$ & $* \leqslant V .7 T$ & 17 \\
\hline $\mathrm{e}-$ & "audience. & re & rq.01 & rq & $\leqslant \wedge .10$ & 1. & Ir.ro & $1 \lambda \varepsilon$ & VO.VY & $* 17.97$ & Tr \\
\hline \multicolumn{12}{|c|}{ The existence of clear definition of responsibilities and specializations of all the employees in the swimming pool. } \\
\hline a- & $\begin{array}{l}\text { "swimming } \\
\text { pool director. }\end{array}$ & 79 & 10.19 & v & $\lambda .7 \leqslant$ & 。 & $7.1 \mathrm{~V}$ & rYT & 9 9т... & $* 9 \wedge . \vee \vee$ & 1 \\
\hline$b-$ & $\begin{array}{l}\text { "deputy } \\
\text { director. }\end{array}$ & $7 V$ & Ar.VT & 7 & $\checkmark . \varepsilon 1$ & $\wedge$ & 9.11 & rrI & 9.90 & $* \wedge \wedge .97$ & 7 \\
\hline c- & "coaches. & 70 & $A \cdot r_{0}$ & v & A. $7 \leqslant$ & 9 & 11.11 & Y1A & ११.४ & $* \wedge \cdot, r$. & $\wedge$ \\
\hline d- & *rescuers. & $7 \leq$ & $\vee 9 .+1$ & ir & $1 \leqslant . \wedge 1$ & $\circ$ & $7.1 \mathrm{~V}$ & YrI & 9.90 & $* \vee 7.97$ & 7 \\
\hline $\mathrm{e}-$ & "workers. & $\leqslant 7$ & $07 . \vee 9$ & rq & ro.A. & 7 & $\mathrm{~V} . \leqslant 1$ & $r+r$ & Ar.ir & $*$ *,$\wedge 0$ & YI \\
\hline \multicolumn{12}{|c|}{ The swimming pool administration hold training courses of crisis management: } \\
\hline a- & "periodical. & 71 & งז.90 & 6 & $V . \Sigma 1$ & v & $1.7 \leq$ & TrT & १1.VV & $* 9 r . \leqslant 1$ & $\varepsilon$ \\
\hline$b-$ & *infrequently. & $r \leq$ & rQ.Tr & 26 & rr.l. & r & rA. YV & 100 & $4 r .89$ & $\cdot .97$ & re \\
\hline
\end{tabular}

Assiut Journal For Sport Science Arts the researcher's meetings with a number of rescue guards, she found lack of satisfaction from their side concerns their salaries, long rescue hours and the inequality between them and the coaches in training courses, or the salaries which result in the occurrence of a crisis; this agrees with the study of (Mohamed Hanafy Soudi) 2006 (9). The administration sees the necessity of scientific qualification of coaches by (53.09\%) of study sample.

6- Results display of study sample responses to the axis phrases "avoiding crises in swimming pools". 
Follow Table (9)

Repetition, percentage, $\mathrm{Ca} 2$ value, and phrases order of study sample responses to axis phrases "avoiding crises in swimming pools" $(\mathrm{N}=81)$

\begin{tabular}{|c|c|c|c|c|c|c|c|c|c|c|c|}
\hline \multirow[t]{2}{*}{ No. } & \multirow[t]{2}{*}{ Phrases } & \multicolumn{2}{|l|}{ agree } & \multicolumn{2}{|c|}{ To some extent } & \multicolumn{2}{|l|}{ disagree } & \multirow{2}{*}{$\begin{array}{l}\text { Estimated } \\
\text { total }\end{array}$} & \multirow{2}{*}{$\begin{array}{l}\text { Relative } \\
\text { weight }\end{array}$} & \multirow[t]{2}{*}{$\mathrm{Ca} 2$} & \multirow[t]{2}{*}{ order } \\
\hline & & repetition & $\%$ & repetition & $\%$ & Repetition & $\%$ & & & & \\
\hline C- & *no courses. & r. & $r v . \cdot \varepsilon$ & 8 & १.१^ & $\leq r$ & or.. 9 & $1 \leqslant 9$ & $\pi . r r$ & *rr. 19 & Tr \\
\hline
\end{tabular}

\begin{tabular}{|c|c|c|c|c|c|c|c|c|c|c|c|}
\hline$a-$ & *water & 7. & $V \leq .+V$ & $1 T$ & 17.0 & $\wedge$ & 9.11 & $r) \leqslant$ & AN. $\cdot V$ & $* 7.97$ & \\
\hline$b-$ & $\begin{array}{l}\text { "equipments } \\
\text { and } \\
\text { machines. }\end{array}$ & 00 & $7 V .9$. & iv & $r \cdot .99$ & 9 & 11.11 & $r \cdot \Lambda$ & 10.7. & $* \leq \varepsilon . V \leq$ & 11 \\
\hline$c-$ & $\begin{array}{l}\text { "official } \\
\text { tournaments. }\end{array}$ & Tr & rᄉ. $\Sigma$. & or & $70 . \leqslant T$ & 0 & 7.18 & 11. & $v \varepsilon . \cdot v$ & $* \leqslant r .07$ & 11 \\
\hline$d-$ & $\begin{array}{l}\text { *friendly } \\
\text { matches. }\end{array}$ & rr & $r V .17$ & $\leqslant 9$ & $7 . \leq 99$ & 1. & Ir.to & $1 v \leq$ & 81.7. & *rq.07 & YV \\
\hline$e-$ & "rescuers. & ו1 & vo.ru & $1 \leq$ & IV.TA & 7 & $V_{.} \leqslant 1$ & TIV & А१. & $* 70, \leqslant 1$ & rq \\
\hline$f-$ & visitors. & $r$. & $r \leq .79$ & or & $70 . \leqslant T$ & $\wedge$ & 9.11 & $1 v \leqslant$ & v1.7. & $* \varepsilon \cdot r r$ & 1. \\
\hline $\mathrm{g}-$ & $\begin{array}{l}\text { conflicting } \\
\text { schedules of } \\
\text { teams. }\end{array}$ & or & $v \cdot r v$ & $1 \leq$ & IV.YA & 1. & ir.ro & $r .9$ & $17 . \cdot 1$ & & rq \\
\hline$h-$ & *other & rt & r. & $\leqslant V$ & $0 \Lambda . r$ & $\wedge$ & 9.11 & 11. & $v \leqslant . v$ & & 17 \\
\hline
\end{tabular}

\begin{tabular}{|c|c|c|c|c|c|c|c|c|c|c|c|}
\hline a- & $\begin{array}{l}\text { "delegated } \\
\text { from board of } \\
\text { directors. }\end{array}$ & 59 & 72.84 & 15 & 18.52 & 7 & 8.64 & 214 & 88.07 & 58.07 & 11 \\
\hline$b-$ & $\begin{array}{l}\text { "senior } \\
\text { coaches. }\end{array}$ & 47 & 58.02 & 26 & 32.10 & 8 & 9.88 & 201 & 82.72 & 28.22 & 22 \\
\hline c- & $\begin{array}{l}\text { "senior } \\
\text { technician. }\end{array}$ & 57 & 70.37 & 15 & 18.52 & 9 & 11.11 & 210 & 68.42 & 50.67 & 15 \\
\hline d- & $\begin{array}{l}\text { "security } \\
\text { officer. }\end{array}$ & 32 & 39.51 & 41 & 50.62 & 8 & 9.88 & 186 & 76.54 & 21.56 & 24 \\
\hline
\end{tabular}

\begin{tabular}{|c|c|c|c|c|c|c|c|c|c|c|c|}
\hline$a-$ & "pool director. & TV & $\Delta r . V T$ & 9 & 11.11 & 0 & 7.18 & YYE & $9 Y .11$ & $* 19.19$ & r \\
\hline$b-$ & "coaches. & 71 & Ar.9o & v & $1.7 \leqslant$ & 7 & $V . \leqslant 1$ & TYE & 94.11 & $* q r . \leqslant 1$ & r \\
\hline $\mathrm{c}-$ & technicians. & ov & $v \cdot . r v$ & iv & $r .999$ & V & $1.7 \leq$ & YIT & NV.rE & $* 01,10$ & ir \\
\hline$d-$ & "workers. & r & rN.rV & $\varepsilon r$ & 01.10 & $\wedge$ & $9 . \wedge \wedge$ & 110 & v. & *rt.r. & ro \\
\hline
\end{tabular}


Follow Table (9)

Repetition, percentage, $\mathrm{Ca} 2$ value, and phrases order of study sample responses to axis phrases "avoiding crises in swimming

\begin{tabular}{|c|c|c|c|c|c|c|c|c|c|c|c|}
\hline \multirow[t]{2}{*}{ No. } & \multirow[t]{2}{*}{ Phrases } & \multicolumn{2}{|l|}{ agree } & \multicolumn{2}{|c|}{ To some extent } & \multicolumn{2}{|l|}{ disagree } & \multirow{2}{*}{$\begin{array}{l}\text { Estimated } \\
\text { total }\end{array}$} & \multirow{2}{*}{$\begin{array}{l}\text { Relative } \\
\text { weight }\end{array}$} & \multirow[t]{2}{*}{$\mathrm{Ca} 2$} & \multirow[t]{2}{*}{ order } \\
\hline & & repetition & $\%$ & repetition & $\%$ & Repetition & $\%$ & & & & \\
\hline \multirow[t]{2}{*}{$\mathrm{C}-$} & *no courses. & $r$. & $r v_{.} \cdot \varepsilon$ & 8 & १.४ & $\leq r$ & or. 9 & $1 \leqslant 9$ & 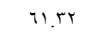 & *rr. 19 & r \\
\hline & $\begin{array}{l}\text { There are } \\
\text { modern } \\
\text { communication } \\
\text { means of } \\
\text { crises } \\
\text { management. }\end{array}$ & $\varepsilon r$ & or. .9 & rq & ro.s. & 9 & 11.11 & 197 & $A \cdot .74$ & *Y. & rT \\
\hline 8 & $\begin{array}{l}\text { In the pool } \\
\text { administration, } \\
\text { there are files } \\
\text { about the past } \\
\text { crises to make } \\
\text { use of them. }\end{array}$ & 01 & $7 r .97$ & ro & $r \cdot . \wedge 7$ & 。 & $7.1 \mathrm{~V}$ & $r \cdot \Lambda$ & 10.7. & $* r q, \leqslant 1$ & 11 \\
\hline 9 & $\begin{array}{l}\text { The } \\
\text { administration } \\
\text { makes a daily } \\
\text { report about } \\
\text { the ratios of } \\
\text { chlorine and } \\
\text { ph. }\end{array}$ & & Ar.vT & $\wedge$ & १.१८ & 7 & $v_{.} \leqslant 1$ & Trt & ११.Vर & $* \wedge$ *.97 & $\varepsilon$ \\
\hline 10 & $\begin{array}{l}\text { The swimming } \\
\text { pool } \\
\text { administration } \\
\text { makes a pre- } \\
\text { scenario of } \\
\text { crises } \\
\text { management. }\end{array}$ & & $v \cdot r v$ & 17 & 19.80 & $\wedge$ & $9 . \wedge \wedge$ & rll & $\Delta \pi . \lambda T$ & $* 01.19$ & $1 \varepsilon$ \\
\hline
\end{tabular}

(Ca2) table value at morale level (0.05) and freedom degree (2) = (5.991)

Table (9) shows that phrases "avoiding crises in

(Ca2) calculated value of study swimming pools" are all sample responses to axis statistically significant at

Assiut Journal For Sport Science Arts 
morale level (0.05), except the two phrases numbered (1 A) and $(3-b)$ where $(\mathrm{Ca} 2)$ calculated value of them were $(0.22, \quad 0.96)$ respectively, which are statistically insignificant value.

As (Ca2) value was between (16.96 - 98.07), and the relative weight was between (61.32 - 93.00\%). Where the phrases numbered (1c, 2a, 2b, 2c, 3a, 4a, 4h, 4f, 5a, 5b, 6a, 6b, 6c, 9, 10) came with high agreement percentage between $(70.37 \%$ : $85.19 \%)$.

The researcher's study assured that there is a clear definition of responsibilities and specializations of workers; as the phase (2a) occupied the first place by $(85.19 \%)$ of the complex director and $82.72 \%$ of his deputy, this agrees with the study of (Sabria Bint Muslim Alyehyay 2006) (5), that the most of the crises management operations are represented in the leadership, as for (Dolan, T, 2006) (14); he referred to the importance of having a crises team in which each person knows what he should do when a crisis occurs, and this is what phrase number (2) confirmed.
The administration also makes periodical courses which is confirmed by phrase (3a) by $(83.95 \%)$ which confirms that the administration is keen on these courses and its importance and it urges workers to join educational courses about crises and how to deal with them; which phrase (6a, or $6 b)$ assured; as it occupied the second place. This contradicts with the results concluded by (Mahmoud Abu Samra, Mohamed Eltity, and Faten Ashour 2012) (10) as they had moderate results on educational programs and courses and guidance in crises for faculties and universities. The researcher sees that there is no pre-made model for the possible crises and how to deal with them especially in official championships and friendly matches, this contradicts with (Adams, Ch. Kritsons,W. 2006) (13); because their study assured that there is no strategic plan to crisis management which contradicts with the results the researcher concluded through the positive axis of avoiding crisis. While (Abdel Hadi Aly Ahmed Excell 2003) (6) agrees with her, about the how far the clubs 
are prepared to athletic crises which include the plan, organization and training, which phrases number $(5 \mathrm{a}, 5 \mathrm{~b}$, and $5 c$ ) confirmed that there is an experienced official to deal with the crisis and he has the authorization from board of administration by $(72.84 \%)$ in addition to the senior coach and the senior technician; which is an integrated team work to face crises which agrees with the study of (Vogelaar. 2005)(17).

The necessity to have a team work to face crises authorized by the higher administration and enjoys the power to take decisions if necessary.

7- Displaying the results of study sample responses to the phrases of axis "the scientific method of crisis management in swimming pools".

\section{Table (10)}

Repetition, percentage, $\mathrm{Ca} 2$ value, and phrases order of study sample responses to axis phrases "the scientific method of crisis management in swimming pools" $(\mathrm{N}=81)$

\begin{tabular}{|c|c|c|c|c|c|c|c|c|c|c|c|}
\hline \multirow[t]{2}{*}{ No. } & \multirow[t]{2}{*}{ phrases } & \multicolumn{2}{|c|}{ agree } & \multicolumn{2}{|c|}{ To some extent } & \multicolumn{2}{|c|}{ disagree } & \multirow{2}{*}{$\begin{array}{c}\text { Estimated } \\
\text { total }\end{array}$} & \multirow{2}{*}{$\begin{array}{l}\text { Relative } \\
\text { weight }\end{array}$} & \multirow[t]{2}{*}{$\mathrm{Ca} 2$} & \multirow[t]{2}{*}{ order } \\
\hline & & repetition & $\%$ & repetition & $\%$ & repetition & $\%$ & & & & \\
\hline 1 & $\begin{array}{c}\text { The swimming } \\
\text { pool } \\
\text { administration } \\
\text { has the } \\
\text { delegation of } \\
\text { authority style } \\
\text { in case of } \\
\text { director's } \\
\text { absence. }\end{array}$ & & $v 9 . .1$ & 1. & ir.ro & v & $1.7 \varepsilon$ & r19 & $9 . .14$ & *VT, r & v \\
\hline $2-$ & $\begin{array}{c}\text { In the } \\
\text { swimming } \\
\text { pool } \\
\text { administration, } \\
\text { there are } \\
\text { cadres have } \\
\text { the ability to } \\
\text { face athletic } \\
\text { crises inside } \\
\text { the swimming }\end{array}$ & TV & AT.YY & 1 & $v . \leqslant 1$ & $\wedge$ & $9 . \wedge 1$ & rr & 9.90 & $* \wedge \Lambda .97$ & r \\
\hline
\end{tabular}


Follow Table (10)

Repetition, percentage, $\mathrm{Ca} 2$ value, and phrases order of study sample responses to axis phrases "the scientific method of crisis management in swimming pools" $(\mathrm{N}=81)$

\begin{tabular}{|c|c|c|c|c|c|c|c|c|c|c|c|}
\hline \multirow[t]{2}{*}{ No. } & \multirow[t]{2}{*}{ phrases } & \multicolumn{2}{|c|}{ agree } & \multicolumn{2}{|c|}{ To some extent } & \multicolumn{2}{|c|}{ disagree } & \multirow{2}{*}{$\begin{array}{c}\text { Estimated } \\
\text { total }\end{array}$} & \multirow{2}{*}{$\begin{array}{l}\text { Relative } \\
\text { weight }\end{array}$} & \multirow[t]{2}{*}{$\mathrm{Ca} 2$} & \multirow[t]{2}{*}{ order } \\
\hline & & repetition & $\%$ & repetition & $\%$ & repetition & $\%$ & & & & \\
\hline 3 & $\begin{array}{l}\text { There are } \\
\text { study and } \\
\text { analysis of all } \\
\text { the conditions } \\
\text { that may be } \\
\text { resulted from } \\
\text { the crisis and } \\
\text { preparation } \\
\text { for it. }\end{array}$ & 01 & vi. & $1 \varepsilon$ & IV.ru & 9 & 11.11 & rll & גז.AT & *or.so & iv \\
\hline 4 & $\begin{array}{l}\text { In the swimming } \\
\text { pool } \\
\text { administration, } \\
\text { there are modern } \\
\text { technological } \\
\text { means of } \\
\text { managing sudden } \\
\text { crises. }\end{array}$ & ON & vi. & 11 & Tr.Tr & 。 & 7.18 & rio & $M . \leqslant \wedge$ & *07.0r & ir \\
\hline 5 & $\begin{array}{c}\text { There are files } \\
\text { for all the } \\
\text { crises related } \\
\text { to the } \\
\text { swimming } \\
\text { pool. }\end{array}$ & $0 \leqslant$ & $77.7 \mathrm{~V}$ & r) & ro.9T & 7 & $v . \leqslant 1$ & r. & $\Delta \tau . \leqslant r$ & $* \varepsilon \leqslant .7 V$ & 11 \\
\hline 6 & $\begin{array}{l}\text { There is a } \\
\text { scientific } \\
\text { method to } \\
\text { analyze } \\
\text { information } \\
\text { and facts } \\
\text { related to the } \\
\text { crisis. }\end{array}$ & 01 & 7 T.97 & r & $r_{\Lambda} \varepsilon$. & v & $1.7 \varepsilon$ & r.T & $\Lambda \varepsilon . Y Y$ & $*$ * . . $\Sigma \varepsilon$ & r. \\
\hline
\end{tabular}


1

\section{Follow Table (10)}

Repetition, percentage, $\mathrm{Ca} 2$ value, and phrases order of study sample responses to axis phrases "the scientific method of crisis management in swimming pools" $(\mathrm{N}=81)$

\begin{tabular}{|c|c|c|c|c|c|c|c|c|c|c|c|}
\hline \multirow[t]{2}{*}{ No. } & \multirow[t]{2}{*}{ phrases } & \multicolumn{2}{|c|}{ agree } & \multicolumn{2}{|c|}{ To some extent } & \multicolumn{2}{|c|}{ disagree } & \multirow{2}{*}{$\begin{array}{c}\text { Estimated } \\
\text { total }\end{array}$} & \multirow{2}{*}{$\begin{array}{l}\text { Relative } \\
\text { weight }\end{array}$} & \multirow[t]{2}{*}{$\mathrm{Ca} 2$} & \multirow[t]{2}{*}{ order } \\
\hline & & repetition & $\%$ & repetition & $\%$ & repetition & $\%$ & & & & \\
\hline \multicolumn{12}{|c|}{ 7-there are reports that may be returned to about the swimming pool in concerning: } \\
\hline a- & $\begin{array}{c}\text { *maintenance } \\
\text { rates. }\end{array}$ & די & $11 . \leqslant 1$ & $v$ & $\wedge .7 \leq$ & $\wedge$ & $9, \wedge \wedge$ & rt. & $9 \cdot .0 \mathrm{r}$ & *^s.or & $\varepsilon$ \\
\hline b- & $\begin{array}{l}\text { *chlorine } \\
\text { ratio in water. }\end{array}$ & $7 \varepsilon$ & $v_{9} \cdot{ }^{\prime} 1$ & $\wedge$ & $9 . \wedge 1$ & 9 & 11.11 & YIV & А१.५. & $* \vee\urcorner . \cdot v$ & 1. \\
\hline c- & $\begin{array}{c}\text { Some } \\
\text { members' } \\
\text { riots. }\end{array}$ & TV & אז.זr & $\leq 9$ & $7 . \leq 99$ & 0 & 7.18 & $111 \varepsilon$ & VO.VY & * ro, 10 & $r \leq$ \\
\hline d- & $\begin{array}{l}\text { The } \\
\text { continuous } \\
\text { absence of } \\
\text { coach or } \\
\text { rescuer. }\end{array}$ & 70 & A. ro & 1. & סז.ri & 7 & $v . \leqslant 1$ & TrI & 9.90 & $* \wedge$. or & r \\
\hline e- & $\begin{array}{l}\text { The } \\
\text { continuous } \\
\text { complaints } \\
\text { from some } \\
\text { employees or } \\
\text { coaches. }\end{array}$ & 01 & $7 r .97$ & $r \leq$ & rq.7 & 7 & $V . \leq 1$ & $r \cdot V$ & 10.19 & $* r \wedge .$. & 19 \\
\hline
\end{tabular}

8- There are pre-made scenarios in the pool administration to deal with the crises related to:

\begin{tabular}{|c|c|c|c|c|c|c|c|c|c|c|c|}
\hline a- & $\begin{array}{l}\text { *equipments } \\
\text { and machines. }\end{array}$ & זי & VY.VA & IT & $1 \leq .11$ & 7 & $v . \leqslant 1$ & r) & $9 . .1 r$ & $* V T . T V$ & v \\
\hline b- & *members. & $\S 1$ & $0.7 \pi$ & Tr & $\varepsilon \cdot V \leq$ & v & $\wedge .7 \leq$ & 197 & A. & $* r r . \leqslant 1$ & rI \\
\hline $\mathrm{C}$ & *coaches. & 7 & vo.ru & ir & $1 \leq . \wedge 1$ & $\wedge$ & $9 . \wedge 1$ & rio & $\wedge \wedge . \leqslant \Lambda$ & * r or or & ir \\
\hline $\mathrm{D}$ & *employees. & rı & $\leq 7.91$ & $r \leq$ & $\leqslant 1.91$ & 9 & 11.11 & 191 & vı. . . & *i人.r. & rt \\
\hline $\mathrm{E}$ & *rescuers. & 00 & $7 V .9$. & YI & ro.9r & 。 & 7.18 & rit & $A v . r \leq$ & $* \varepsilon \wedge, r$. & 17 \\
\hline $\mathrm{F}$ & *other. & $\varepsilon$ & $\leq q,{ }^{\prime} \wedge$ & ro & $\varepsilon r . r \mid$ & 7 & $V . \leqslant 1$ & 197 & $1 . .77$ & $* r \leqslant .97$ & rI \\
\hline
\end{tabular}

9-when a crisis occurs in the swimming pool, it is dealt with using:

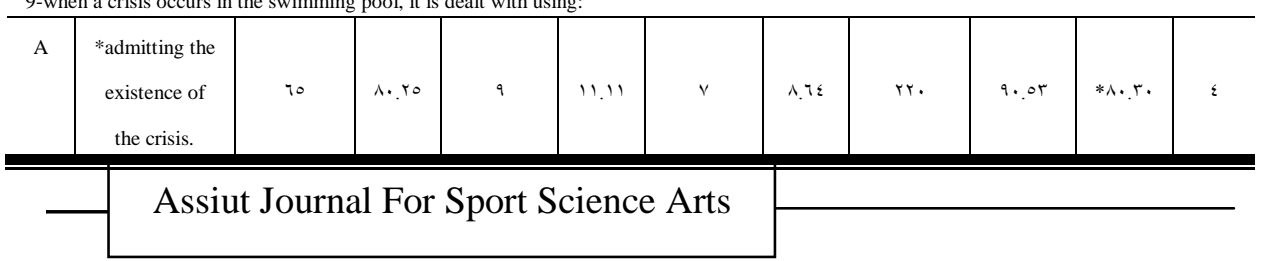


Follow Table (10)

Repetition, percentage, $\mathrm{Ca} 2$ value, and phrases order of study sample responses to axis phrases "the scientific method of crisis management in swimming pools" $(\mathrm{N}=81)$

\begin{tabular}{|c|c|c|c|c|c|c|c|c|c|c|c|c|}
\hline \multirow[t]{2}{*}{ No. } & \multirow[t]{2}{*}{ phrases } & \multicolumn{3}{|c|}{ agree } & \multicolumn{2}{|c|}{ To some extent } & \multicolumn{2}{|c|}{ disagree } & \multirow{2}{*}{$\begin{array}{c}\text { Estimated } \\
\text { total }\end{array}$} & \multirow{2}{*}{$\begin{array}{l}\text { Relative } \\
\text { weight }\end{array}$} & \multirow[t]{2}{*}{$\mathrm{Ca} 2$} & \multirow[t]{2}{*}{ order } \\
\hline & & \multicolumn{2}{|c|}{ repetition } & $\%$ & repetition & $\%$ & repetition & $\%$ & & & & \\
\hline B & $\begin{array}{l}\text { *determining } \\
\text { crisis reasons. }\end{array}$ & \multicolumn{2}{|c|}{$7 \leqslant$} & vq. 1 & 9 & 11.11 & $\wedge$ & $9 . \wedge 1$ & イ^ & 19.1 & $* \vee\urcorner . \vee \vee$ & 9 \\
\hline $\mathrm{C}$ & $\begin{array}{l}\text { *determining } \\
\text { who is } \\
\text { responsible } \\
\text { for the crisis } \\
\text { occurrence. }\end{array}$ & \multicolumn{2}{|c|}{ Tr } & V7. $0 \leqslant$ & 1. & ortro & 9 & 11.11 & Yo & $\Lambda \Lambda . \wedge$ & $* \neg \wedge . \vee$ & ir \\
\hline $\mathrm{D}$ & $\begin{array}{l}\text { *quickly } \\
\text { solving the } \\
\text { crisis. }\end{array}$ & \multicolumn{2}{|c|}{79} & 10.19 & $v$ & $\Lambda . T \leq$ & 。 & $7.1 \mathrm{~V}$ & דוצי & qт... & $* 9 \wedge . \vee \vee$ & 1 \\
\hline $\mathrm{E}$ & $\begin{array}{c}\text { *ignoring the } \\
\text { crisis. }\end{array}$ & \multicolumn{2}{|c|}{ rt } & r..01 & 7 & $V . \leqslant 1$ & $\leq r$ & or. 9 & 101 & Tr. & *หา. $\vee \leqslant$ & ro \\
\hline \multicolumn{13}{|c|}{ 10-when a crisis occurs, you make use of experts to solve it: } \\
\hline A & \multicolumn{2}{|c|}{$\begin{array}{c}\text { *maintenance } \\
\text { companies (in case of } \\
\text { crisis related to } \\
\text { equipments). }\end{array}$} & $7 \varepsilon$ & $\vee १ . \cdot 1$ & 11 & 15.01 & 7 & $v_{\text {. }} \leqslant 1$ & rr. & $9.0 \%$ & *VT. or & $\varepsilon$ \\
\hline B & \multicolumn{2}{|c|}{$\begin{array}{l}* \text { members of board } \\
\text { directors (in case of } \\
\text { crisis related to } \\
\text { members). }\end{array}$} & 74 & $V T .0 \leq$ & ir & $1 \leq .11$ & v & $\Lambda .7 \leqslant$ & TIV & ง . . . & * १人. or & 1. \\
\hline $\mathrm{c}$ & \multicolumn{2}{|c|}{$\begin{array}{l}* \text { members of Game } \\
\text { Federation (in case of } \\
\text { crisis related to clubs). }\end{array}$} & 7. & $V \leqslant . \cdot V$ & ir & 17.0 & $\wedge$ & $9 . \wedge 1$ & $Y \leq$ & $\Lambda \Lambda \cdot \cdot v$ & $* 7.97$ & 10 \\
\hline
\end{tabular}

(Ca2) table value at morale level (0.05) and freedom degree $(2)=(5.991)$

Table (10) shows that

(Ca2) calculated value of study sample responses to axis phrases "the scientific method of crisis management in swimming pools" are all statistically significant at morale level (0.05).

$$
\text { As }(\mathrm{Ca} 2) \text { value was }
$$
between (18.30 - 98.07), and the relative weight was between $(75.72 \%$ - 93.00\%);

Assiut Journal For Sport Science Arts 
where the phrases number $(1$, 2, 3, 4, 7a, 7b, 7d, 8a, 8c, 9a, 9b, 9c, 9d, 10a, 10b, and 10c) came with high agreement percentage between $(71.60 \%$ : 85.19).

From what is mentioned previously, it is clear that the high agreement percentage which confirms that swimming complexes use the scientific method of crisis management, as the swimming pool administration enjoys the style of delegation of authority in case of the director's absence which should be prepared and trained on before. This is due to the existence of cadres that have the ability of confrontation; which was confirmed by phrase number (2) by $(82.72 \%)$ that occupied the second place in the axis for its importance and positivity. This is sustained by the modern technological means used by the administration in solving crises; which was confirmed by phrase number (4) by $(71.60 \%)$. Also, there is a followed style and steps when the crisis occurs; we find phrases number (9a, 9b, 9c, 9d) when a crisis occurs, we must admit its occurrence, determine its reasons and who is responsible for it and to quickly solve it. In addition, the complex administration has the cadres that enjoy the ability to face crises; this phrase occupied the second place by $(82.72 \%)$. This agrees with the study of (Edert, $\mathbf{R}$ and Griffin, R. 2005) (15) in ways of crisis management, also when the crisis occurs, we should use the specialized experts in the crisis kind; this was confirmed by phrases (10a, 10b, 10c) which agrees with the study of (Mohamed Elserafy 2005) (8) the methodological administration in dealing with crises in light of preparation, knowledge, understanding and the available potentials, the skills and the prevailing patterns of administration. It also has premade scenarios to deal with these crises. But the researcher sees that the pre-made scenario to deal with the members occupies the $21^{\text {st }}$ place by $(50.62 \%)$ which is a tiny percentage for a number of members and the difference in cultural \& knowledge level of crises management in swimming pools, the axis in general depends on the scientific method of crises management and this disagrees with the study of Sbria Bin 
Muslim Alyehewy (2006) (5) that concluded that most of crises management operations are concentrated on the higher leadership and not distributed.
9- Displaying the study sample responses to the phrases of axis "evaluation method of administration performance in swimming pools".

Table (11)

Repetition, percentage, $\mathrm{Ca} 2$ value, and phrases order of study sample responses to axis phrases "evaluation method of administration performance in swimming pools" ( $\mathrm{N}=81)$

\begin{tabular}{|c|c|c|c|c|c|c|c|c|c|c|c|}
\hline \multirow[t]{2}{*}{ No. } & \multirow[t]{2}{*}{ Phrases } & \multicolumn{2}{|c|}{ agree } & \multicolumn{2}{|c|}{ To some extent } & \multicolumn{2}{|c|}{ disagree } & \multirow{2}{*}{$\begin{array}{l}\text { Estimated } \\
\text { total }\end{array}$} & \multirow{2}{*}{$\begin{array}{l}\text { Relative } \\
\text { weight }\end{array}$} & \multirow[t]{2}{*}{$\mathrm{Ca} 2$} & \multirow[t]{2}{*}{ order } \\
\hline & & repetition & $\%$ & repetition & $\%$ & repetition & $\%$ & & & & \\
\hline 1 & $\begin{array}{c}\text { There are } \\
\text { periodical } \\
\text { and official } \\
\text { records } \\
\text { about the } \\
\text { swimming } \\
\text { pool } \\
\text { accredited } \\
\text { by club } \\
\text { director } \\
\text { and board } \\
\text { of directors } \\
\text { about the } \\
\text { pool } \\
\text { activity. }\end{array}$ & or & $70.2 \pi$ & 19 & YT.\&Y & 9 & 11.11 & r. T & $\Lambda \leq . \vee V$ & $*$ * $9 . \leqslant 1$ & ri \\
\hline 2 & $\begin{array}{l}\text { There are } \\
\text { annual } \\
\text { reports } \\
\text { about the } \\
\text { crises that } \\
\text { occurred in } \\
\text { the } \\
\text { swimming } \\
\text { pool. }\end{array}$ & r. & $v \varepsilon . \cdot V$ & 18 & 19.80 & • & $\because .1 \mathrm{~V}$ & riv & A..r. & *q. & $\wedge$ \\
\hline 3 & $\begin{array}{l}\text { There are } \\
\text { daily } \\
\text { reports } \\
\text { about the } \\
\text { ratio of } \\
\text { chemicals } \\
\text { used in the } \\
\text { swimming } \\
\text { pool. }\end{array}$ & 01 & Tr.97 & $r \varepsilon$ & ra.7r & 1 & $v_{.} \leqslant 1$ & $r \cdot v$ & 10.19 & $* \Gamma \Lambda_{.}$. & r. \\
\hline 4 & $\begin{array}{c}\text { There are } \\
\text { a map }\end{array}$ & $\because$ & $v 0 . r_{1}$ & 14 & 17.00 & $v$ & $1.7 \leqslant$ & rit & $\wedge \wedge . \wedge q$ & $* ฯ \varepsilon . \wedge 9$ & 1. \\
\hline
\end{tabular}




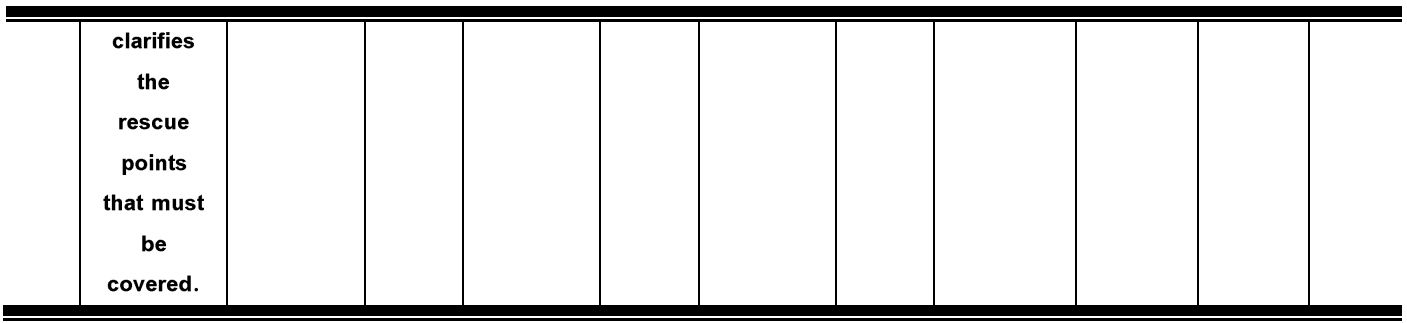

Follow Table (11)

Repetition, percentage, $\mathrm{Ca} 2$ value, and phrases order of study sample responses to axis phrases "evaluation method of administration performance in swimming pools" $(\mathrm{N}=81)$

\begin{tabular}{|c|c|c|c|c|c|c|c|c|c|c|c|}
\hline \multirow[t]{2}{*}{ No. } & \multirow[t]{2}{*}{ Phrases } & \multicolumn{2}{|c|}{ agree } & \multicolumn{2}{|c|}{ To some extent } & \multicolumn{2}{|c|}{ disagree } & \multirow{2}{*}{$\begin{array}{c}\text { Estimated } \\
\text { total }\end{array}$} & \multirow{2}{*}{$\begin{array}{c}\text { Relative } \\
\text { weight }\end{array}$} & \multirow[t]{2}{*}{$\mathrm{Ca} 2$} & \multirow[t]{2}{*}{ order } \\
\hline & & repetition & $\%$ & repetition & $\%$ & repetition & $\%$ & & & & \\
\hline 5 & $\begin{array}{l}\text { There are } \\
\text { steps to be } \\
\text { followed to } \\
\text { treat crises } \\
\text { in the } \\
\text { swimming } \\
\text { pool. }\end{array}$ & 01 & 97.97 & rr & rV.17 & $\wedge$ & $9 . \wedge \wedge$ & r. O & $\Lambda \varepsilon . r q$ & * & rr \\
\hline 6 & $\begin{array}{l}\text { There is a } \\
\text { map } \\
\text { clarifies the } \\
\text { performance } \\
\text { schedules } \\
\text { of athletic } \\
\text { teams. }\end{array}$ & 4t & $V 7.0 \leqslant$ & 1. & or.tro & 9 & 11.11 & rlo & $\wedge \wedge . \leqslant \wedge$ & $* \uparrow \Lambda . \cdot \vee$ & ir \\
\hline 7 & $\begin{array}{l}\text { To have } \\
\text { ways to } \\
\text { punish the } \\
\text { responsible } \\
\text { for the } \\
\text { crisis } \\
\text { occurrence. }\end{array}$ & 7. & $V \varepsilon . \cdot V$ & 17 & 19.80 & 。 & $7.1 V$ & YIV & ᄉ ..r. & $* \neg$ *.V & $\wedge$ \\
\hline 8 & $\begin{array}{l}\text { To have } \\
\text { ways to } \\
\text { reward who } \\
\text { helped to } \\
\text { end the } \\
\text { crisis. }\end{array}$ & $\varepsilon r$ & or.. 9 & re & r9.01 & 1 & $V . \leqslant 1$ & 199 & $\wedge 1 . \wedge 9$ & *Y . V & rT \\
\hline
\end{tabular}

9-there are forms for the continuous evaluation of performance in the swimming pool that covers all the employees in it: 


\begin{tabular}{|c|c|c|c|c|c|c|c|c|c|c|c|}
\hline A & $\begin{array}{l}{ }^{*} \text { pool } \\
\text { director. }\end{array}$ & 79 & 10.19 & 7 & $v . \& 1$ & 7 & V.\&1 & rro & 94.09 & $* q \wedge . \ldots$ & 1 \\
\hline B & $\begin{array}{l}{ }^{*} \text { deputy } \\
\text { director. }\end{array}$ & 77 & $\wedge 1 . \leqslant \wedge$ & $\wedge$ & $9 . \wedge \wedge$ & $\vee$ & 1. $7 \varepsilon$ & rri & $9 . .90$ & $* \Lambda \varepsilon . O r$ & - \\
\hline C & ${ }^{*}$ employees. & rr & ra.01 & $\varepsilon r$ & $0{ }^{4} .9$ & 7 & $v . \leqslant 1$ & $1 \wedge \wedge$ & vV.rv & $* Y\urcorner . V \varepsilon$ & ro \\
\hline$E$ & "rescuers. & 09 & VY.^E & it & 17.0 & 9 & 11.11 & rir & AV.Y & $* 0 \vee .19$ & iv \\
\hline $\mathbf{F}$ & * other. & rr & +9.01 & $\varepsilon \varepsilon$ & OE.rY & 0 & 7.18 & 119 & $\vee \vee . \vee \wedge$ & *ץ 9.04 & rE \\
\hline
\end{tabular}

Follow Table (11)

Repetition, percentage, $\mathrm{Ca} 2$ value, and phrases order of study

sample responses to axis phrases "evaluation method of

administration performance in swimming pools" $(\mathrm{N}=81)$

\begin{tabular}{|c|c|c|c|c|c|c|c|c|c|c|c|}
\hline \multirow[t]{2}{*}{ No. } & \multirow[t]{2}{*}{ Phrases } & \multicolumn{2}{|c|}{ agree } & \multicolumn{2}{|c|}{ To some extent } & \multicolumn{2}{|c|}{ disagree } & \multirow{2}{*}{$\begin{array}{c}\text { Estimated } \\
\text { total }\end{array}$} & \multirow{2}{*}{$\begin{array}{c}\text { Relative } \\
\text { weight }\end{array}$} & \multirow[t]{2}{*}{$\mathrm{Ca} 2$} & \multirow[t]{2}{*}{ order } \\
\hline & & repetition & $\%$ & repetition & $\%$ & repetition & $\%$ & & & & \\
\hline
\end{tabular}

10-there is a scientific method to evaluate the employees' performance in the swimming pool:

\begin{tabular}{|c|c|c|c|c|c|c|c|c|c|c|c|}
\hline A & $\begin{array}{l}{ }^{*} \text { pool } \\
\text { director. }\end{array}$ & 11 & Ar.qo & v & $1.7 \leqslant$ & 1 & $v . \leqslant 1$ & PYE & 94.11 & *ar. $\{1$ & $r$ \\
\hline B & $\begin{array}{l}\text { "deputy } \\
\text { director. }\end{array}$ & r & $\vee \vee . \vee \wedge$ & ir & $1 \leq . \wedge 1$ & 1 & $v . \leqslant 1$ & P19 & $9 . .14$ & *VY.TV & $v$ \\
\hline C & $\begin{array}{l}{ }^{*} \text { employees } \\
\text { of systems. }\end{array}$ & 11 & $v 0 . r 1$ & ir & 17.0 & $\mathrm{v}$ & $1.7 \leqslant$ & Y17 & $\wedge \wedge . \wedge 9$ & $* ฯ \leqslant . \wedge q$ & 1. \\
\hline D & "rescuers. & 00 & IV.9. & 11 & rT.YT & $\wedge$ & $9 . \wedge \wedge$ & $r . q$ & 1... & $* \leqslant 0 . \leqslant 1$ & 11 \\
\hline $\mathrm{E}$ & ${ }^{*}$ coaches. & 11 & AT.90 & $v$ & $1.7 \leq$ & 1 & $v . \leqslant 1$ & YYE & $9 \varphi .11$ & $* a r . \leqslant 1$ & $r$ \\
\hline
\end{tabular}

11-there are a scientific method to measure the crisis management operation:

\begin{tabular}{|c|c|c|c|c|c|c|c|c|c|c|c|}
\hline A & $\begin{array}{l}{ }^{*} \text { crisis } \\
\text { nature. }\end{array}$ & 70 & ט. & 11 & 1r.01 & 。 & 7.18 & rrt & 91.49 & $* \wedge \cdot . \wedge q$ & $\varepsilon$ \\
\hline B & $\begin{array}{c}\text { *time of } \\
\text { occurrence. }\end{array}$ & 10 & A.,Yo & 1. & Ir.ro & 1 & V.\&1 & $r r$ & 9.90 & $* \Lambda . .0 r$ & 0 \\
\hline C & $\begin{array}{c}\text { *its } \\
\text { persons. }\end{array}$ & $\Delta \Lambda$ & vi.t. & 17 & 19.80 & $v$ & $\Lambda .7 \varepsilon$ & rir & AV.To & $* 0 \leqslant . \wedge 9$ & 17 \\
\hline D & $\begin{array}{l}\text { *factors } \\
\text { resulted } \\
\text { from it. }\end{array}$ & 11 & vo.ru & ir & $|\leq . \wedge|$ & $\wedge$ & $৭ . \wedge \wedge$ & rio & $\Lambda \wedge . \& \Lambda$ & * & Ir \\
\hline$E$ & $\begin{array}{l}{ }^{*} \text { crisis } \\
\text { result. }\end{array}$ & 11 & vo.ru & 11 & $1 r .01$ & 9 & 11.11 & r $1 \varepsilon$ & $\wedge \wedge . \cdot \vee$ & * & 10 \\
\hline$F$ & $\begin{array}{c}\text { *the } \\
\text { decision } \\
\text { about it. }\end{array}$ & 71 & Ar.qo & $\wedge$ & $9 . \wedge \wedge$ & 。 & 7.18 & rro & $q 4.0 q$ & *q & 1 \\
\hline
\end{tabular}

Ca2) table value at morale level $(0.05)$ and freedom degree $(2)=(5.991)$ 
Table (11) shows that (Ca2) calculated value of study sample responses to axis phrases "evaluation method of administration performance in swimming pools" are all statistically significant at morale level (0.05).

(Ca2) value was between (26.74 - 98.00), and the relative weight was between $(77.37 \%$ - 92.59\%), where the phrases number $(2,4,6,7,9 \mathrm{a}$, 9b, 9d, 9h, 10a, 10b, 10c, 11a, $11 \mathrm{~b}, 11 \mathrm{c}, 11 \mathrm{~d}, 11 \mathrm{~h}, 11 \mathrm{y})$ came with high agreement percentage between $(71.60 \%$ $85.19 \%)$.

The study made by the researcher confirmed that the axis of evaluation method of administration performance is a result of the axis of scientific method of crises management, as the all axis' phrases were positive and of high percentage whereas there are annual reports about the crises that occurred in the swimming pool, and the agreement percentage on phrase number (2) was $(74.07 \%)$ and it occupies the eighth place for its importance in analyzing the problem and knowing its reasons, which agrees with the study of (Abdel Hadi Aly
Ahmed Excell 2003) (6) their expectations of crisis repetition, the time of its occurrence and how far they are prepared for it. Also, the existence of a map that shows the rescue points and performance times of athletic teams which facilitates evaluating the administration performance during the crisis, this was clarified by phrases number (4, and 6) by percentage $(75.31 \%, 76.54 \%)$. Moreover, there is firmness when the crisis occurs, as this phrase obtained high agreement percentage $(74.07 \%)$. What emphasizes that the swimming complexes follow modern evaluation methods to evaluate administration performance is that they have performance evaluation form continuously for all the employees (director, deputy director, coaches, workers and rescuers) that enjoy high percentage between $(75.31 \%-85.19 \%)$. They also have scientific methods and techniques for evaluation, which all the axis phrases confirmed; phrases number (11a, 11b, 11c, 11d, 11h, 11y) agree with the study of (Kelsay, L. 2007) (16) that the crisis' nature, timing and 
persons affect measuring crisis management operation, and consequently, affect the consequential results and decisions as phrase number (11y) obtained high percentage $(83.95 \%)$ which indicates that decision is the most important step in the operation of crisis management measuring. That disagrees with the study of (Sherif Mohamed Awad 2007) (4) that decision makers are the high and medium categories, while (Dolan. 2006) (14) sees the importance of having a team group in time of crises where every person knows what he should do in time of crisis occurrence. The researcher sees that decision making is influenced by continuous change, intervention, impulse and complication, so the research praises and recommends teams of crises management for its experience and knowledge of crises management, its solution, and taking decision without reference to high categories as it is a waste of time.

\section{Research conclusions:}

In light of research questions and the statistical procedures, the researcher concluded the following:
First: reasons of crises occurrence in swimming pools:

- Lack of coordination between the swimming pool administration and the technical systems.

- Lack of coordination between the swimming pool administration and the athletic activity administration.

- Lack of sufficient scientific qualification of maintenance workers in swimming pool.

- Lack of scientific qualification of rescue in swimming pool.

- $\quad$ Lack of alternative plans to use them when rescuers are absent.

- Lack of regulations to deal with rescuers.

- Not following the scientific method of water sterilization systems inside the pool.

Second: Avoiding crises in swimming pool complexes:

- The existence of clear definition of responsibilities and specializations of all the employees in the swimming pool (director - deputy director - coaches - rescuers).

- The swimming pool administration should make 
training courses of crises management periodically.

To have a clear definition of responsibilities and specializations of all the employees in the complex.

- The administration must urge the employees to have educational courses about crises and how to deal with them.

- To have a booklet of instructions and guidance for rescuers on the swimming pool.

Third: the scientific method of crises management in swimming pools:

- The administration should had the style of delegation of authority when the director absence from the swimming complex.

- The administration should have cadres that enjoy the ability of facing athletic crises inside the swimming complex.

- To have reports about maintenance rates and chlorine ratios in water to be referred to.

To have reports about coaches and rescuers absence this helps in accountability when the crisis occurs.

- To deal with the crisis using the method of (acknowledgment determining reasons

determining who is responsible

- quickly solving it).

To make use of experts in order to solve the crisis when it occurs.

Fourth: evaluation methods of administration performance in swimming pools:

- To have annual reports about the crises that occurred.

- To have a map that shows rescue points which have to be covered and the timing of teams' performance on the swimming pool.

- To have method of punishing the careless people when a crisis occurs.

- To have a scientific method for continuous evaluation of the employees' performance.

- To use the scientific method in measuring the crisis management concerning (crisis' nature - time of its occurrence - its persons - the consequential factors - crisis result - and the decision taken about it).

\section{Recommendation:}

1- $\quad$ To use the questionnaire made by the researcher to measure the level of crises management in swimming pools. 
2- $\quad$ To make similar studies on clubs in different governorates in order to recognize the scientific method of crises management.

3- To perform similar studies on clubs of low financial level in order to recognize the level of scientific method of crises management.

4- To make a study comparing between clubs of high financial resources (elite clubs) and those of low financial resources in order to recognize the aspects of agreement and disagreement among them in the scientific method of crises management.

References:

First: Arabic References:

1- Ismail Hamed Othman (1998): the crisis of playground riots; a time bomb, Helwan University, Faculty of Physical Education for males, an unpublished study.

\section{2- Ashraf Mahmoud Hussein}

Elogeily (2004): the economic crises in the non-governmental athletic authorities, a published Ph. D., Faculty of Physical Education for males in Haram, Helwan University, Cairo.

\section{3- Anwar Wagdy Elwakeel} (2003): an article about - the necessary confrontations of playground riots crisis, Faculty of Physical Education, Almenya University, 2003.

4- Sherif Hamed Mohamed Moawad (2007): taking the administrative decision to face some crises in ministry of youth, an unpublished master thesis, Helwan University, Cairo.

\section{5- Sabria Bint Muslin} Elyehyawy (2006): crises management in governmental intermediate schools in Almadina Almonawra, Educational Sciences \& Islamic Studies Magazine, Issue 18, p. 1- 49.

6- Abel Mahdy Aly Ahmed Excell (2003): the reality of managing some administrative crises in athletic clubs in Bahrain Kingdom, an unpublished master thesis, Bahrain University.

7- Aziz Araas Sabty (2002): crises management in business organizations in the Jordan industrial sector, Arbid, Jordan. 8- Mohamed Elserafy (2003): modern administrative 
conceptions, $1^{\text {st }}$ version, Aldar Elelmia, Amman.

9- Mohamed Hanafy Mahoud Seoudi (2006): evaluating the general banking policies in the field of financial crises management with an empirical study on the two crises of currency and recession in Egypt, an unpublished master thesis, Cairo University, Cairo.

10- Mahmoud Abu Samra, Mohamed Eltity, Faten Ashuor (2012): crises management of physical education departments and its circles in the Palestinian high education institutes from their students point of view, AlAzhar magazine in Gaza, series of Humanitarian sciences, vol. 14 , issue 1, p. $35-72$.

\section{1- Mo'men Saied Abdel} Allah Soliman (2012): the effectiveness of swimming pools directors in athletic crises management, an unpublished master thesis, Meoufia University, Al-Sadat City.

12- Wagih Mohamed Abdel Allah Nada (2000): designing some preparation models to face the expected athletic crises in Egypt, an unpublished $\mathrm{Ph}$. D. thesis, Faculty of Physical Education for males, Helwan University, 2000.

Second: Foreign References: 1 r-Adams, ch,kritsons ,w (2006) . AN Analysis of secondary schooL 's crisis management preparedness, national implications, national gournai for publishing and mentoring doctoral student . vol. 1 ,n. 1, p 1,7.

I \&-DOLAN , T .(2006).Few schools Are ready to manage ACRISIS - THE Education Digest. Vol72,N.2,P4,8. 10 -Edert, $\mathbf{R}$ and Griffin, $R$. (2005). Business Essentials. Fifth Edition, Upper Saddle River, New York.

1 7-Kelsay, L. (2007). After math of crisis, how colleges respond to prospective students. Journal of college Admission. N.197, P 6-13.

iv-Vogelaar, R. (2005). Rhetorical Approaches to Crises Communication: The Research, Development and Validation of an Image Repair Situational Theory for Assiut Journal For Sport Science Arts 
Educational and Leaders, Saint Dissertations).

Louis University. (Doctoral 